\title{
A Logistic Regression Model for The Prediction of HBV-Related Cirrhosis
}

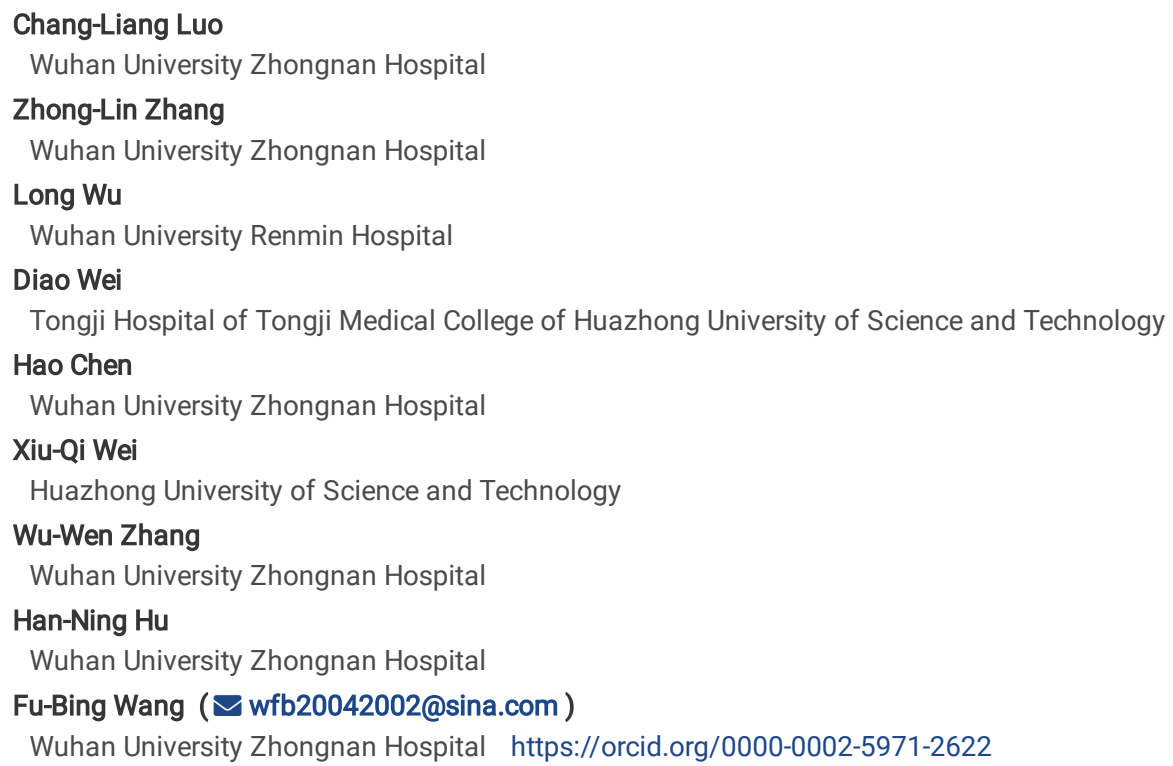




\section{Abstract}

Background: Cirrhosis is one of the most severe complications at the late stage of chronic HBV infection. The liver biopsy is the gold standard for the diagnosis of liver cirrhosis. However, a liver biopsy is associated with the risk of severe complications and a high cost. It is therefore necessary to find several biomarkers for the diagnosis of HBV-related cirrhosis.

Methods: The research was proceeded to evaluate the diagnostic value of hematological parameters to find the surrogate markers in HBV-related cirrhosis. The research was proceeded on the training set, which was recruited from Zhongnan Hospital, including 102 HBV-related cirrhosis and 102 healthy individuals. The levels of selected hematological parameters were analyzed. The receiver operating characteristic curves were generated to evaluate the diagnostic effectiveness of these parameters. A logistic regression model was built and validated using four validation sets consisting of 261 patients.

Results: The result show that the level of RDW, MPV, MPV/PC ratio, PLR and NLR were all significantly higher in HBV-related cirrhosis patients compared to healthy individuals. Most of these parameters owned a moderate AUC in HBV-related cirrhosis patients. However, their diagnostic sensitivities or specificities were unsatisfactory. Therefore, a logistic regression model was built by combining these hematological parameters. The model showed great diagnostic value with the AUC of 0.987 , sensitivity of $96.1 \%$ and specificity of $95.1 \%$. Besides, the other four validation sets were generated to validate the logistic regression model and all showed good AUC with moderate specificities and sensitivities.

Conclusions: The data indicate that the model might be substantially useful for the diagnosis of HBV-related cirrhosis.

\section{Introduction}

Cirrhosis is one of the most severe complications of chronic HBV infection especially at the late stage. The 1-year mortality rate of patients with cirrhosis varies from $1 \%$ (early-stage cirrhosis) to $57 \%$ (decompensated cirrhosis) ${ }^{[1-3]}$.In addition, the 5 -year cumulative hepatocellular carcinoma risk is $10 \%-17 \%$ in cirrhotic patients ${ }^{[4]}$. Therefore, early diagnosis of HBV-related cirrhosis is crucial for early therapy and good prognosis ${ }^{[5-7]}$.

The diagnosis of liver cirrhosis is traditionally based on the patients' manifestations, laboratory assays and imaging tests. However, these diagnostic methods have their limitations. Although the liver biopsy is the gold standard for cirrhosis diagnosis, it is invasive, difficult, expensive, and sometimes accompanied by significant morbidity ${ }^{[8,9]}$. It is therefore not feasible for all patients. It is necessary to find biomarkers which can distinguish HBV-related cirrhosis in a noninvasive, accurate, and simple manner. Former studies have suggested numerous biochemical molecules such as aspartate aminotransferase, alanine aminotransferase and gamma globulin for cirrhosis or liver fibrosis detection ${ }^{[10-13]}$. However, most of these proteins were investigated in patients with chronic hepatitis $\mathrm{C}$.

It is reported that chronic inflammation is associated with persistent hepatic injury and concurrent tissue regeneration which leads to sequential development of fibrosis, cirrhosis and eventually HCC ${ }^{[11]}$.In addition, about one fourth of mild chronic hepatitis patients are affected by cirrhosis within 1 to 13 years of hepatitis onset ${ }^{[14]}$.

Considering the role of inflammatory responses in cirrhosis development, we designed a research to evaluate diagnostic value of hematological parameters in HBV-related cirrhosis patients compared to healthy people. We focused on white blood cell count (WBC), red blood cell distribution width (RDW), mean platelet volume (MPV), MPV/PC ratio, platelet count/lymphocytes ratio (PLR) and number of neutrophils/lymphocytes ratio (NLR). The corresponding ROC curves were used to evaluate diagnostic value. At last, a model was established for the prediction of HBV-related cirrhosis and was validated in four different validation sets from four different hospitals.

\section{Materials And Methods}

\section{Training set}

We investigated 102 patients with HBV-related cirrhosis and 102 healthy individuals at Zhongnan Hospital from November 2016 to March 2018 . The retrospectively study was under approval of Medical Ethics Committee, Zhongnan Hospital of Wuhan University (201707), and written informed consent was obtained from all participants. Their demographic and blood test results were reviewed in the hospital medical database.

\section{Logistic regression models}

A formula for predicting HBV-related cirrhosis was built based on the data in the Training set.-The standard logistic regression formula is:

Logit $(P)=\beta 0+\beta 1 X 1+\beta X 2+\ldots \ldots+\beta n X n$.

Regarding Logit $(P)=\ln [p /(1-p)]$, " $p$ " is the estimated probability of HBV-related cirrhosis, " $\beta 0$ " is a constant " $\beta$ " is the influence coefficient, " $n$ " is the number of influence factors and " $\mathrm{X}$ " is the influence factors.

\section{Validation sets}

Four validation sets were recruited from four centers (Zhongnan Hospital of Wuhan University ;Tongji Hospital, Tongji Medical College of Huazhong University Science and Technology; Union Hospital, Tongji Medical College of Huazhong Science and Technology University; Renmin Hospital of Wuhan University) and were used to assess the performance of the model. They included $261 \mathrm{HBV}$-related cirrhosis patients and 288 healthy people. $70 \mathrm{HBV}$-related cirrhosis patients 
and 80 healthy people, $35 \mathrm{HBV}$-related cirrhosis patients and 45 healthy people, $31 \mathrm{HBV}$-related cirrhosis patients and 38 healthy people, $125 \mathrm{HBV}$-related cirrhosis patients and 125 healthy people were recruited in these sets, respectively.

\section{Statistical analysis}

Statistical analysis was performed using SPSS version 22.0 (SPSS, Chicago, IL, USA) or Prism6 (GraphPad software, La Jolla, CA). Data were presented as the mean \pm standard deviation (SD). The Shapiro-Wilk test was used to check the normality of the distribution. Normally distributed numeric variables were evaluated by Student's t-test or one-way analyses of variance (ANOVA). Non-normally distributed variables were analyzed by the Mann-Whitney test or nonparametric test and Chi-square test was used to analyze the categorical variables. A difference was considered statistically significant when $P<0.05$. The area under the ROC curve was measured to evaluate the diagnostic value of each hematological parameter.

\section{Results}

\section{Demographic parameters of the training set}

129 patients with cirrhosis were recruited in this -research. The flow chart of the research was showed in Figure 1. After exclusion of 16 patients with alcoholic cirrhosis and 11 patients with chronic hepatitis $C$ infection, 102 patients with HBV-related cirrhosis and 102 age-matched healthy people were enrolled. The healthy people had no medical record of tumor and matched the HBV-related cirrhosis patients in gender $(p=0.389)$, age $(p=0.572)$, height $(p=0.646)$, weight $(p=0.190)$ and smoking $(p=0.751)$ (Table S1).

\section{Hematological parameters of the training set}

We focused on the expression level of WBC, RDW, MPV, MPV/PC ratio, PLR, and NLR in cirrhosis patients. As presented in Table 1, the expression level of RDW, MPV, MPV/PC ratio, PLR and NLR were all significantly higher in HBV-related cirrhosis patients compared to healthy individuals. No significant difference in WBC was found between - HBV-related cirrhosis patients and healthy people. ROC curve analysis showed that the AUC of RDW, MPV, MPV/PC ratio, PLR and NLR were $0.970,0.772,0.876,0.702$ and 0.817 in the cirrhosis patient group (Figure 2A). The information of diagnostic value of RDW, MPV, MPV/PC ratio, PLR and NLR was listed in Table $\mathbf{2} 2$

\section{The correlation between clinical parameters and hematological parameters}

Chronic inflammation is associated with persistent hepatic injury and concurrent tissue regeneration, leading to sequential development of fibrosis, cirrhosis and eventually Hepatocellular carcinoma. We analyzed the correlation between clinical parameters and hematological parameters to find out the role of these parameters in the progression and metastasis of HBV-related cirrhosis patients. The result showed MPV/PC ratio and PLR were significantly correlated with the Child-Pugh score while other parameters not (Figure 2B to 2F). In addition, all these hematological parameters RDW, MPV, MPV/PC ratio, PLR and NLR were not correlated with smoking or gender (Table 2).

\section{The logistic regression model for HBV-related cirnhosis}

The parameters that were significantly changed in the HBV-related cirrhosis patients were then used in the multivariate logistic regression model. RDW, MPV, MPV/PC ratio, PLR and NLR were all considered as independent variables (Table 1) and were -used for the building of multivariate model. The data from 102 HBV-related cirrhosis patients and 102 healthy volunteers in the training set was used to build the logistic regression model. The resultant model for predicting HBV-related cirrhosis was:

Logit $(P)=29.167-0.109(M P V)-1.571(R D W)-73.438(M P V / P C$ ratio)-0.045(NLR)- 0.011 (PLR), the Classification Table show that the Predicted Percentage Correct is $94.6 \varangle$,and the degree of freedom(df) was $8(p=0.847)$, suggesting that the model fitted well. The diagnostic value of the model was good with the AUC of 0.987 (Figure $2 A$ ) and the estimated probability at specificity and sensitivity maximum sum are at a cut-off probability of 0.774 , which means if the estimated probability was $<0.774$ was defined as the HBV-related cirrhosis patient group. On the contrary, those with a probability of $\geq 0.774$ would be defined as the negative group.

\section{Multicenter validation of the model}

The validity of the model was assessed in four different validation sets. $261 \mathrm{HBV}$-related cirrhosis patients $(70,35,31$ and 125 patients from four centers, respectively) and 288 healthy volunteers $(80,45,38$ and 125 volunteers from four centers, respectively) were enrolled for the research.

The estimated probability of $261 \mathrm{HBV}$-related cirrhosis patients and 228 healthy volunteers were calculated by using the formula Logit (P). In the cohort of Zhongnan Hospital, the probability of 60 (out of 70) HBV-related cirrhosis patients were less than 0.774 , and the probability of 70 (out of 80 ) healthy volunteers were more than 0.774 (Figure $3 \mathrm{~A}$ ). The AUC of the model for HBV-related cirrhosis was 0.866 , with sensitivity of $85.7 \%$ and specificity of $87.5 \%$, respectively. In the cohort of Tongji Hospital, the probability of 28 (out of 35) HBV-related cirrhosis patients were less than 0.774 , and the probability of 42 (out of 45 ) healthy volunteers were more than 0.774 (Figure $3 B$ ). The AUC of the model for HBV-related cirrhosis was 0.880 , with sensitivity of $80.0 \%$ and specificity of $93.3 \%$, respectively. In the cohort of Union Hospital, the probability of 24 (out of 31 ) HBV-related cirrhosis patients were less than 0.774 , and the probability 33 (out of 39) healthy volunteers were more than 0.774 (Figure 3 C). The AUC of the model for HBV-related cirrhosis was 0.813 , with sensitivity of $77.4 \%$ and specificity of $84.6 \%$, respectively .In the cohort of Renmin Hospital, the probability 110 (out of 125) HBV-related cirrhosis patients were less than 0.774 , and the probability 113 (out of 125) healthy volunteers were more than 0.774 (Figure 3D). The AUC of the model for HBV-related cirrhosis was 0.892 , with sensitivity of $88.0 \%$ and specificity of $90.4 \%$, respectively . The diagnostic performances of this were presented in Table 3 . 


\section{Discussion}

Early diagnosis of cirrhosis is important for reducing the complications in patients with chronic HBV infection ${ }^{[15,16]}$.The liver biopsy is the standard test for distinguishing different liver diseases such as cirrhosis ${ }^{[15]}$ but this test is often associated with high risks of other severe complications, as well as patient discomfort, and costly expense. Therefore, identification a reliable and non-invasive cirrhosis diagnosis method is in high demand by physicians and surgeons.

In the present research, we attempted to build a novel affordable and effective model to predict HBV-related cirrhosis using hematological parameters. The blood test, which is routinely done to the chronic HBV infection patients, has essential implications for the natural history of chronic HBV infection ${ }^{[17]}$. We evaluated the diagnostic value of hematological parameters including WBC, RDW, MPV, MPV/PC ratio, PLR and NLR. Our results indicate that the expression level of RDW, MPV, MPV/PC ratio, PLR and NLR were significantly increased in HBV-related cirrhosis patients. Most of them were indicative of HBV-related cirrhosis. Among them, RDW owned the best diagnostic value (AUC=0.970) for HBV-related cirrhosis. However, no significant correlation was found between RDW and the Child-Pugh score. RDW, which is used in the differential diagnosis of anemia, is a measure of the range of variation of red blood cell volume. Previous studies have reported that high RDW level was related to the high mortality risk of in in patients with various disorders ${ }^{[18-20]}$.In our study, we found that RDW was profoundly higher in HBV-related cirrhosis patients compared to healthy people. Our data is consistent with the previous report ${ }^{[21]}$. The reasons of the elevated RDW might be vitamin B12 or iron deficiency. Interestingly, the AUC of MPV/PC ratio was lower than RDW, but MPV/PC ratio had the highest specificity for HBV-related cirrhosis, in addition, MPV/PC ratio and PLR were significantly correlated with the Child-Pugh score, while RDW not. Therefore, it would be better that combining multiple parameters to detect HBV-related cirrhosis.

We built the model which combining multiple hematological parameters including RDW, MPV, MPV/PC ratio, PLR and NLR. It had better diagnostic value than any single hematological parameter. Furthermore, we validated the logistic regression model in four different validation sets. We recruited HBV-related cirrhosis patients and healthy volunteers from four different hospitals to constitute different validation sets to avoid the selection bias. The model showed good diagnostic value in all validation sets, indicating that it is effective in predicting HBV-related cirrhosis patients. Previous studies have reported the important role of single biochemical indicators or hematological parameters in cirrhosis diagnosis. Kayadibi ${ }^{[22]}$ showed that cirrhosis might be accurately predicted through measuring platelet count, ALT and AST. Koda $\mathrm{M}^{[23]}$ also found that AST to platelet ratio could be a surrogate marker for cirrhosis detection. However, most of these indices were tested in HCV-related cirrhosis patients. In addition, the effectiveness of combinatory inflammatory index in the diagnosis of HBV-related cirrhosis is not well studied. In our research, the model we built showed a better predictive ability than any single inflammatory index. Therefore, this model could be a promising tool for the diagnosis of HBV-related cirrhosis.

\section{Conclusion}

This new logistic regression model we built might improve the diagnosis of HBV-related cirrhosis.

\section{Abbreviations}

RT-qPCR: Real-time quantitative PCR PLR: platelet count/lymphocytes ratio

NLR: number of neutrophils/lymphocytes ratio (NLR)

\section{Declarations}

\section{Ethics approval and consent to participate}

The retrospectively study was under approval of Medical Ethics Committee, Zhongnan Hospital of Wuhan University (201707), and written informed consent was obtained from all participants

\section{Consent for publication}

Not applicable

\section{Acknowledgements}

Not applicable

\section{Declaration of conflicting interest}

The authors declare that there are no conflicts of interest.

\section{Availability of data and materials}

The datasets used and analyzed during the current study are available from the corresponding author on reasonable request.

\section{Funding}


This work was supported by Science and Technology Innovation Fostering Foundation of Zhongnan Hospital of Wuhan University (cxpy20160025), and National Natural Science Foundation of China (No. 81672114). This work also was supported by Hubei Province health and family planning scientific research project (WJ2018H0011).

\section{Author Contributions}

Chang-Liang Luo and Zhong-Lin Zhang performed the study; Chang-Liang Luo and Hao Chen analyzed the data; Wu-Wen Zhang, Long Wu区Diao Wei and XiuQi Wei were responsible for collecting the sample and clinical data; Fu-Bing Wang, Han-Ning Hu and Chang-Liang Luo designed the study and wrote the manuscript; Fu-Bing Wang was responsible for obtaining funding; Chang-Liang Luo and Zhong-Lin Zhang are joint first authors; Fu-Bing Wang and Han-Ning Hu are joint corresponding authors.

\section{References}

1. Arvaniti V, D'Amico G, Fede G, Manousou P, Tsochatzis E, Pleguezuelo M, et al. Infections in patients with cirrhosis increase mortality four-fold and should be used in determining prognosis. Gastroenterology. 2010;139(4):1246-56, 56.e1-5.

2. D'Amico G, Garcia-Tsao G, Pagliaro L. Natural history and prognostic indicators of survival in cirrhosis: a systematic review of 118 studies. Journal of hepatology. 2006;44(1):217-31.

3. Fede G, D'Amico G, Arvaniti V, Tsochatzis E, Germani G, Georgiadis D, et al. Renal failure and cirrhosis: a systematic review of mortality and prognosis. Journal of hepatology. 2012;56(4):810-8.

4. Fattovich G, Bortolotti F, Donato F. Natural history of chronic hepatitis B: special emphasis on disease progression and prognostic factors. Journal of hepatology. 2008;48(2):335-52.

5. [EASL clinical practice guidelines. Management of chronic hepatitis B]. Gastroenterologie clinique et biologique. 2009;33(6-7):539-54.

6. Cox TR, Erler JT. Molecular pathways: connecting fibrosis and solid tumor metastasis. Clinical cancer research : an official journal of the American Association for Cancer Research. 2014;20(14):3637-43.

7. Lok AS, McMahon BJ. Chronic hepatitis B: update 2009. Hepatology (Baltimore, Md.). 2009;50(3):661-2.

8. Munteanu M. Non-invasive biomarkers FibroTest-ActiTest for replacing invasive liver biopsy: the need for change and action. Journal of gastrointestinal and liver diseases : JGLD. 2007;16(2):173-4.

9. Sebastiani G, Alberti A. Non invasive fibrosis biomarkers reduce but not substitute the need for liver biopsy. World journal of gastroenterology. 2006;12(23):3682-94.

10. Gines P, Quintero E, Arroyo V, Teres J, Bruguera M, Rimola A, et al. Compensated cirrhosis: natural history and prognostic factors. Hepatology (Baltimore, Md.). 1987;7(1):122-8.

11. Kwon OS, Choi SH, Kim JH. [Inflammation and Hepatic Fibrosis, Then Hepatocellular Carcinoma]. The Korean journal of gastroenterology = Taehan Sohwagi Hakhoe chi. 2015;66(6):320-4.

12. Park GJ, Lin BP, Ngu MC, Jones DB, Katelaris PH. Aspartate aminotransferase: alanine aminotransferase ratio in chronic hepatitis $C$ infection: is it a useful predictor of cirrhosis? Journal of gastroenterology and hepatology. 2000;15(4):386-90.

13. Wai CT, Greenson JK, Fontana RJ, Kalbfleisch JD, Marrero JA, Conjeevaram HS, et al. A simple noninvasive index can predict both significant fibrosis and cirrhosis in patients with chronic hepatitis C. Hepatology (Baltimore, Md.). 2003;38(2):518-26.

14. Raoufy MR, Vahdani P, Alavian SM, Fekri S, Eftekhari P, Gharibzadeh S. A novel method for diagnosing cirrhosis in patients with chronic hepatitis B: artificial neural network approach. Journal of medical systems. 2011;35(1):121-6.

15. Lefkowitch JH. Liver biopsy assessment in chronic hepatitis. Archives of medical research. 2007;38(6):634-43.

16. Poon RT, Fan ST, Lo CM, Liu CL, Ng IO, Wong J. Long-term prognosis after resection of hepatocellular carcinoma associated with hepatitis B-related cirrhosis. Journal of clinical oncology : official journal of the American Society of Clinical Oncology. 2000;18(5):1094-101.

17. Wang J, Yan X, Yang Y, Chang H, Jia B, Zhao XA, et al. A novel predictive model using routinely clinical parameters to predict liver fibrosis in patients with chronic hepatitis B. Oncotarget. 2017;8(35):59257-67.

18. Ani C, Ovbiagele B. Elevated red blood cell distribution width predicts mortality in persons with known stroke. Journal of the neurological sciences. 2009;277(1-2):103-8.

19. Dabbah S, Hammerman H, Markiewicz W, Aronson D. Relation between red cell distribution width and clinical outcomes after acute myocardial infarction. The American journal of cardiology. 2010;105(3):312-7.

20. Sen HS, Abakay O, Tanrikulu AC, Sezgi C, Taylan M, Abakay A, et al. Is a complete blood cell count useful in determining the prognosis of pulmonary embolism? Wiener klinische Wochenschrift. 2014;126(11-12):347-54.

21. Huang R, Yang C, Wu K, Cao S, Liu Y, Su R, et al. Red cell distribution width as a potential index to assess the severity of hepatitis B virus-related liver diseases. Hepatology research : the official journal of the Japan Society of Hepatology. 2014;44(14):E464-70.

22. Kayadibi H, Yasar B, Ozkara S, Serdar MA, Kurdas OO, Gonen C. The diagnostic accuracy of the Forns index, platelet count and AST to Platelet Ratio Index derived fibrosis index for the prediction of Hepatitis $\mathrm{C}$ virus-related significant liver fibrosis and cirrhosis. Scandinavian journal of clinical and laboratory investigation. 2014;74(3):240-7.

23. Koda M, Matunaga Y, Kawakami M, Kishimoto Y, Suou T, Murawaki Y. Fibrolndex, a practical index for predicting significant fibrosis in patients with chronic hepatitis C. Hepatology (Baltimore, Md.). 2007;45(2):297-306.

Page 5/13 
Tables

Table1 Hematological parameters analysis of Training set

\begin{tabular}{|llll|}
\hline Parameters & \multicolumn{1}{l}{ HBV-related cirrhosis } & Healthy controls & P \\
\cline { 2 - 3 } & $\mathrm{n}=102$ & $\mathrm{n}=102$ & \\
\hline WBC & $6.46 \pm 3.61$ & $6.18 \pm 1.26$ & 0.115 \\
\hline MPV (fL) & $9.71 \pm 1.20$ & $8.59 \pm 0.97$ & $<0.0001$ \\
\hline RDW( $\mathbb{}$ ) & $17.68 \pm 3.64$ & $13.07 \pm 0.64$ & $<0.0001$ \\
\hline MPV/PC ratio & $0.086 \pm 0.046$ & $0.041 \pm 0.012$ & $<0.0001$ \\
\hline NLR & $6.45 \pm 7.43$ & $1.83 \pm 0.73$ & $<0.0001$ \\
\hline PLR & $229.61 \pm 170.06$ & $114.99 \pm 40.73$ & $<0.0001$ \\
\hline
\end{tabular}

Hematological parameters are expressed as mean \pm SD; WBC: White blood cell, PC: platelet count, MPV: mean platelet volume, MPV: mean platelet volume, RDW: red blood cell distribution width, MPV/PC ratio: mean platelet volume to platelet count ratio, NLR: neutrophil to lymphocyte ratio, PLR: platelet to lymphocyte ratio;

Table 2 Correlation between hematological parameters level and clinical parameters

\begin{tabular}{|c|c|c|c|c|c|c|c|c|c|c|c|c|}
\hline \multirow[t]{2}{*}{ Parameters } & \multirow[t]{2}{*}{ Group } & \multirow[t]{2}{*}{$n$} & \multicolumn{2}{|l|}{ MPV } & \multicolumn{2}{|l|}{ RDW } & \multicolumn{2}{|l|}{ MPV/PC ratio } & \multicolumn{2}{|l|}{ NLR } & \multicolumn{2}{|l|}{ PLR } \\
\hline & & & $\begin{array}{l}\text { Mean } \\
\pm S D\end{array}$ & $\begin{array}{l}P \\
\text { value }\end{array}$ & Mean \pm SD & $\begin{array}{l}P \\
\text { value }\end{array}$ & Mean \pm SD & $P$ value & $\begin{array}{l}\text { Mean } \\
\pm S D\end{array}$ & $\begin{array}{l}P \\
\text { value }\end{array}$ & Mean \pm SD & $\begin{array}{l}P \\
\text { value }\end{array}$ \\
\hline \multirow[t]{2}{*}{ Sex } & Male & 73 & $9.70 \pm 1.23$ & 0.596 & $17.43 \pm 3.15$ & 0.899 & $0.085 \pm 0.046$ & 0.251 & $5.93 \pm 6.79$ & 0.795 & $220.69 \pm 158.73$ & 0.750 \\
\hline & Female & 29 & $9.72 \pm 1.12$ & & $18.30 \pm 4.65$ & & $0.087 \pm 0.047$ & & $7.74 \pm 8.83$ & & $252.08 \pm 196.99$ & \\
\hline \multirow[t]{2}{*}{ Smoking } & Negative & 74 & $9.75 \pm 1.18$ & 0.260 & $17.81 \pm 3.51$ & 0.793 & $0.083 \pm 0.043$ & 0.804 & $5.95 \pm 6.71$ & 0.689 & $231.14 \pm 159.56$ & 0.477 \\
\hline & Positive & 28 & $9.61 \pm 1.25$ & & $17.35 \pm 4.02$ & & $0.093 \pm 0.052$ & & $7.77 \pm 9.06$ & & $225.60 \pm 198.32$ & \\
\hline \multirow[t]{2}{*}{$\begin{array}{l}\text { Child-Pugh } \\
\text { score }\end{array}$} & $A-B$ & 74 & $9.74 \pm 1.15$ & 0.632 & $17.69 \pm 3.95$ & 0.549 & $0.077 \pm 0.041$ & $0.001^{\star *}$ & $6.94 \pm 8.36$ & 0.848 & $246.27 \pm 162.36$ & $0.016^{\star}$ \\
\hline & $\mathrm{C}$ & 28 & $9.63 \pm 1.33$ & & $17.66 \pm 2.71$ & & $0.109 \pm 0.051$ & & $5.16 \pm 3.84$ & & $185.59 \pm 184.80$ & \\
\hline
\end{tabular}

$\star p<0.05, * * p<0.01$

Table 3Diagnostic performances of Logistic regression model in training set and validation sets

\begin{tabular}{|llllll|}
\hline \multirow{2}{*}{ Group } & \multicolumn{5}{l}{ HBV-related cirrhosis patients vs Healthy controls } \\
& AUC & $95 \% \mathrm{Cl}$ & P value & Se (\%) & Sp (\%) \\
\hline Training set & & & & & \\
\hline Zhongnan Hospital & 0.987 & $0.975-0.999$ & $<0.0001$ & 96.1 & 95.1 \\
\hline Validation sets & & & & & \\
\hline Zhongnan Hospital & 0.866 & $0.803-0.929$ & $<0.0001$ & 85.7 & 87.5 \\
\hline Tongji Hospital & 0.880 & $0.797-0.963$ & $<0.0001$ & 80.0 & 93.3 \\
\hline Union Hospital & 0.813 & $0.705-0.920$ & $<0.0001$ & 77.4 & 84.6 \\
\hline Renmin Hospital & 0.892 & $0.847-0.937$ & $<0.0001$ & 88.0 & 90.4 \\
\hline
\end{tabular}

Se: sensitivity ; Sp: specificity

\section{Figures}




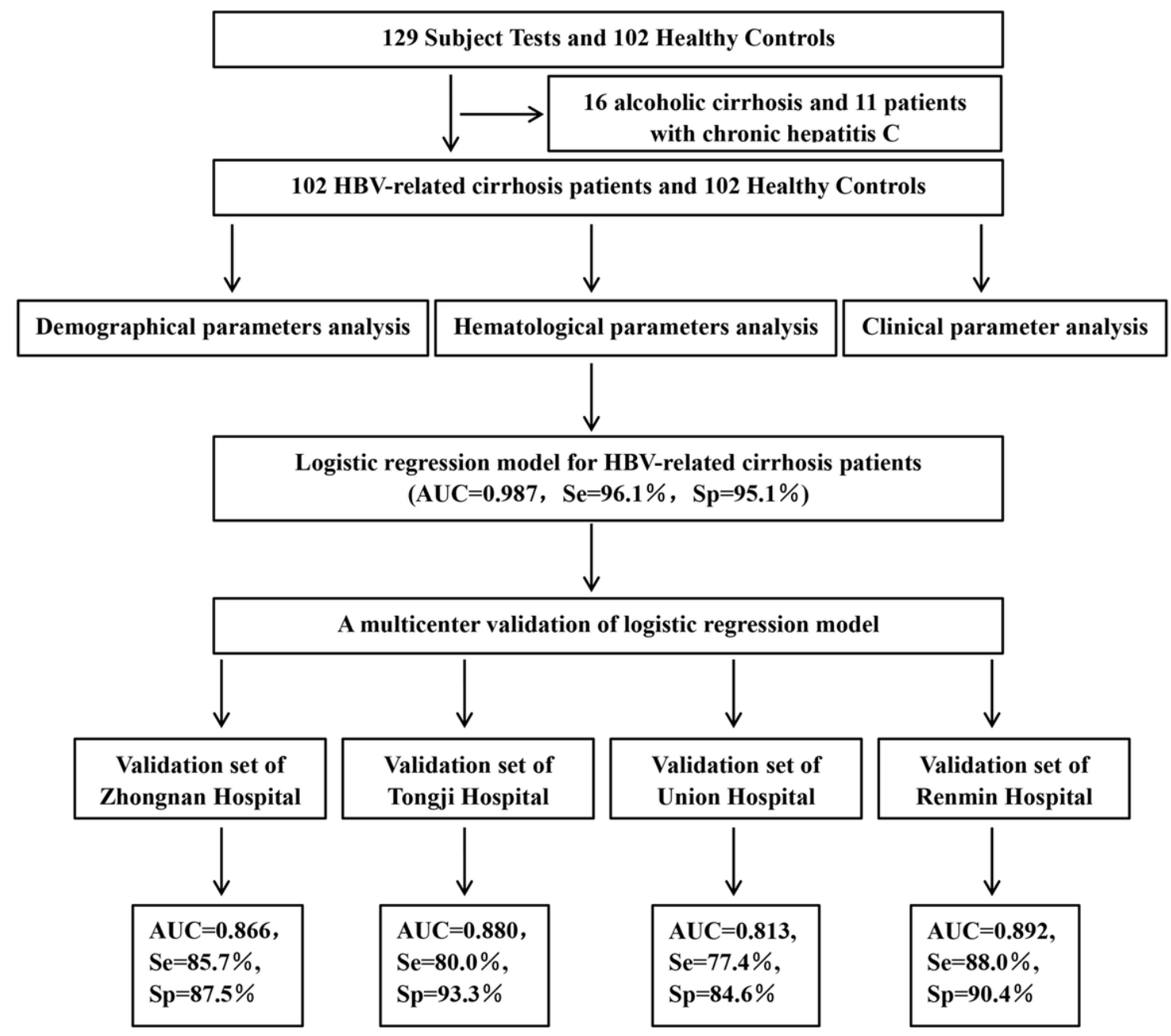

Figure 1

The flow chart of the retrospective study. 


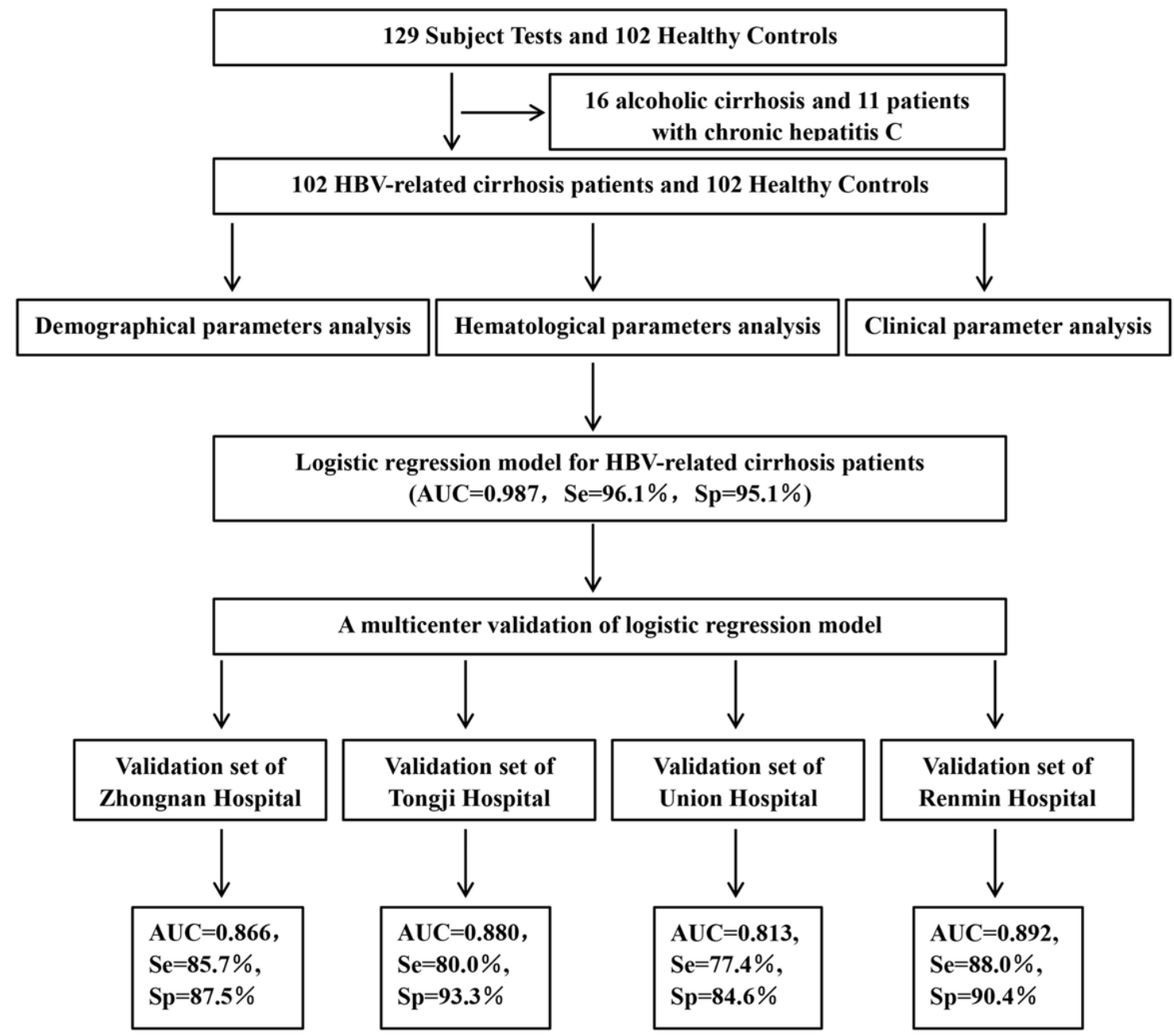

Figure 1

The flow chart of the retrospective study. 


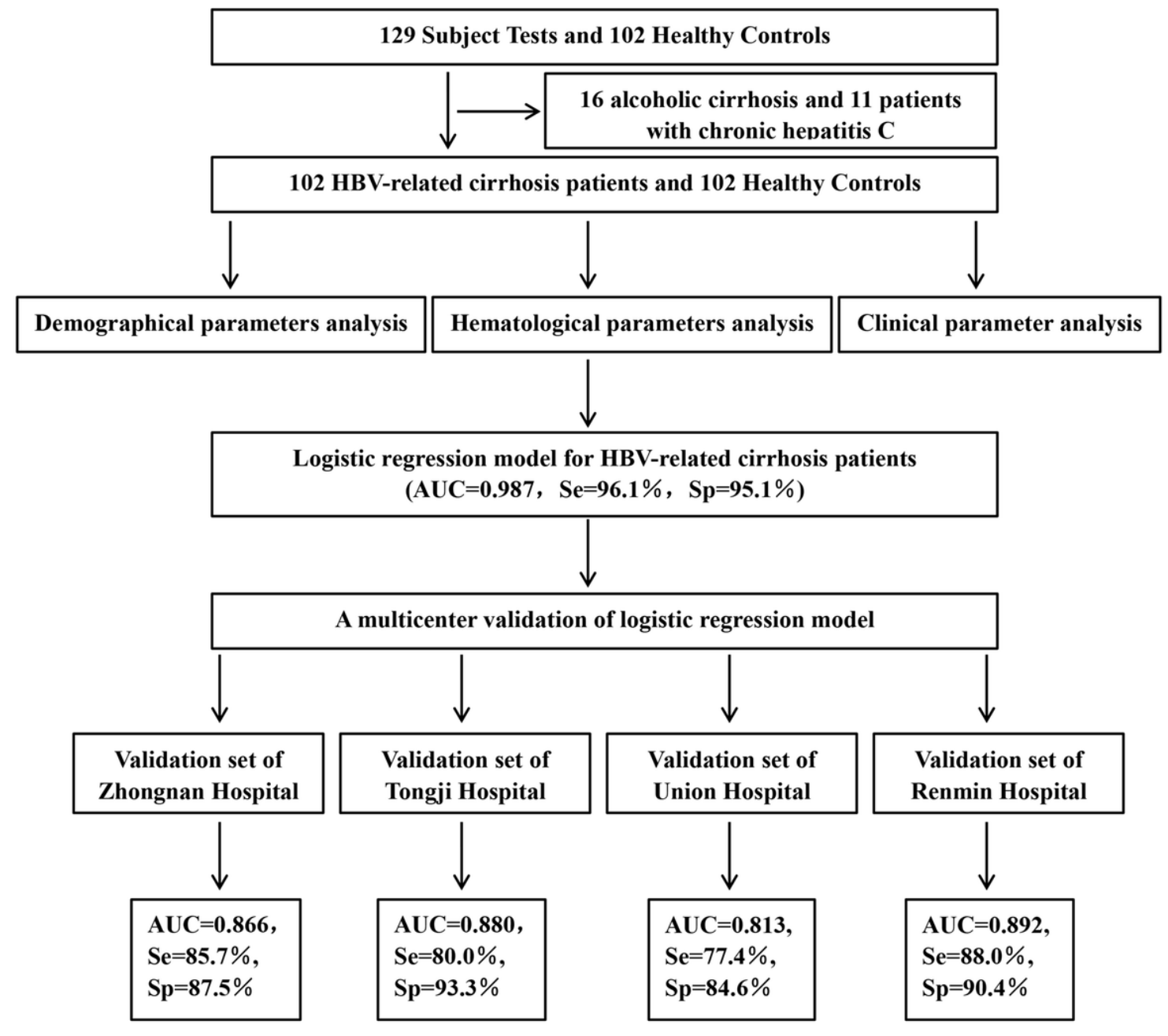

Figure 2

The flow chart of the retrospective study.

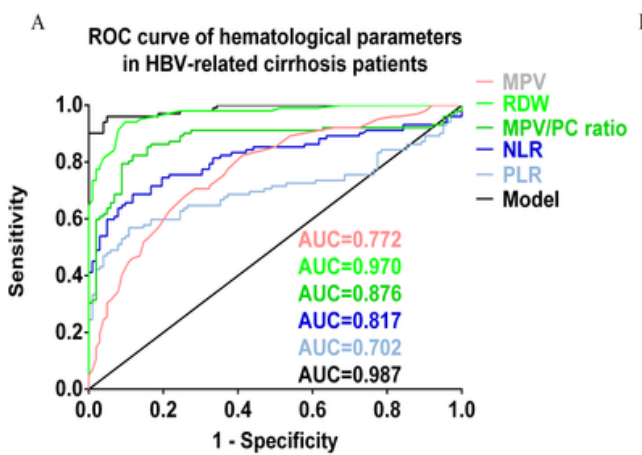

D

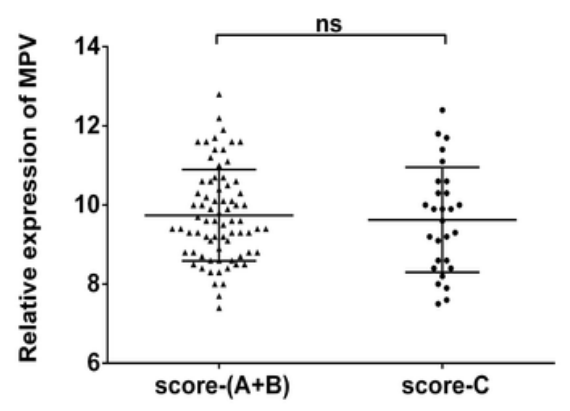

B

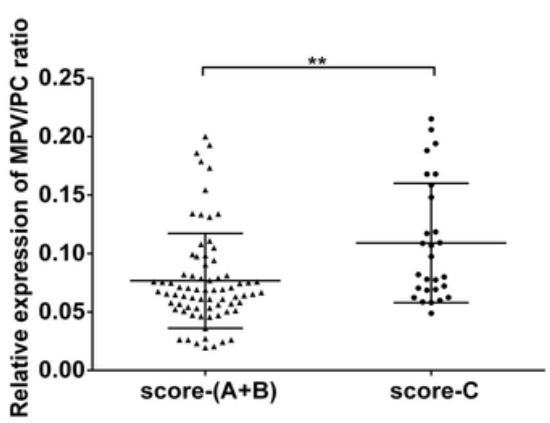

E

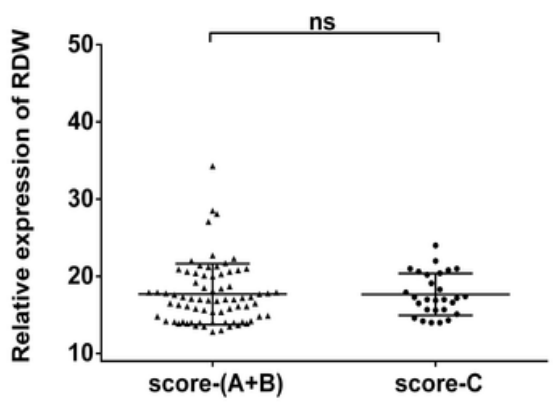

C

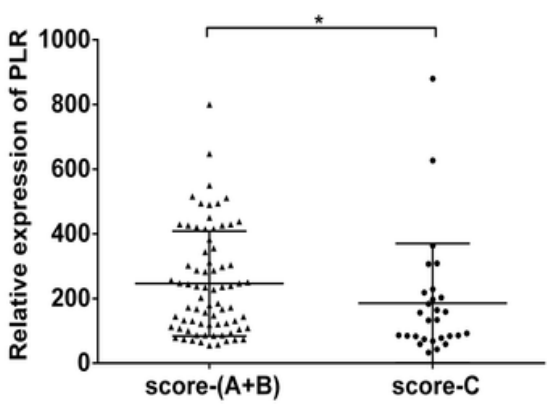

F

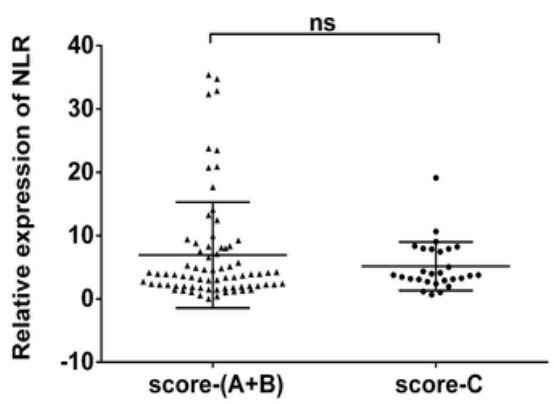


Figure 3

(A) The ROC curve analysis for the diagnostic value of MPV (AUC $=0.772,95 \% \mathrm{Cl}=0.708-0.836, p<0.0001), \mathrm{RDW}(\mathrm{AUC}=0.970,95 \% \mathrm{Cl}=0.949-0.990, \mathrm{p}<$ $0.0001), \mathrm{MPV} / \mathrm{PC}$ ratio $(\mathrm{AUC}=0.876,95 \% \mathrm{Cl}=0.822-0.931, \mathrm{p}<0.0001), \mathrm{NLR}(\mathrm{AUC}=0.817,95 \% \mathrm{Cl}=0.755-0.879, \mathrm{p}<0.0001), \mathrm{PLR}(\mathrm{AUC}=0.702,95 \% \mathrm{Cl}=$ $0.626-0.778, \mathrm{p}<0.0001)$ and model $(\mathrm{AUC}=0.987,95 \% \mathrm{Cl}=0.975-0.999, \mathrm{p}<0.0001)$ in 102 patients with HBV-related cirrhosis.. (B) MPV/PC ratios of patients with HBV-related cirrhosis in different Child-Pugh scores $(p=0.001)$. (C) PLR of patients with HBV-related cirrhosis in different Child-Pugh scores ( $p=0.016)$. ( $)$ MPV of patients with HBV-related cirrhosis in different Child-Pugh scores ( $p=0.632)$. (E) RDW of patients with HBV-related cirrhosis in different Child-Pugh scores $(p=0.549)$. (F) NLR of patients with HBV-related cirrhosis in different Child-Pugh scores $(p=0.848)$. ns \no significance, ${ }^{*} p<0.05,{ }^{* *} p<0.01$.

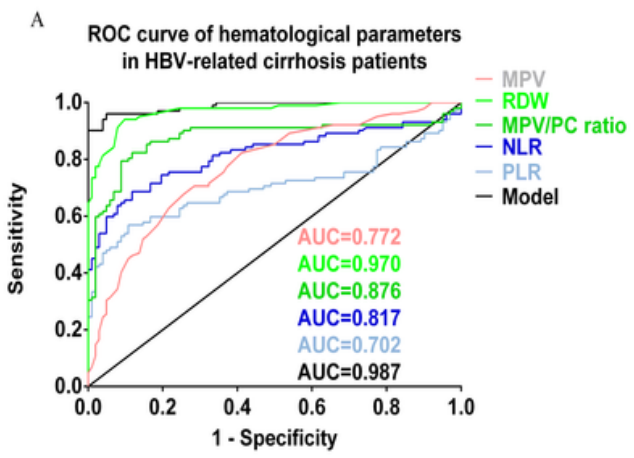

D

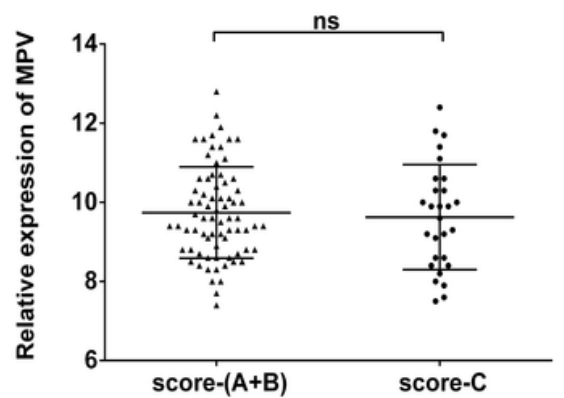

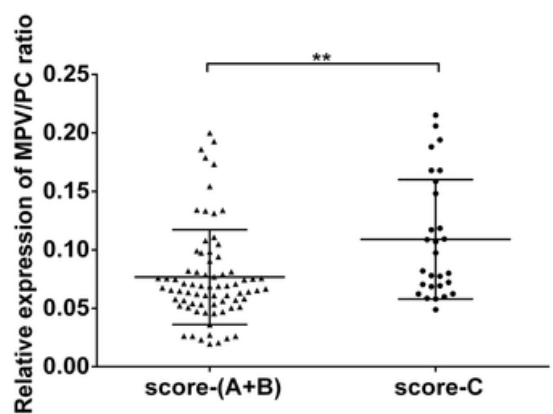

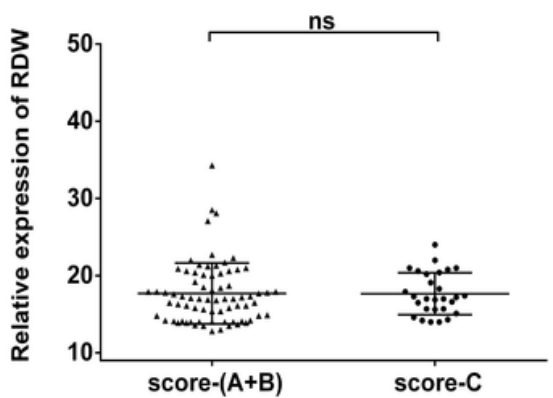

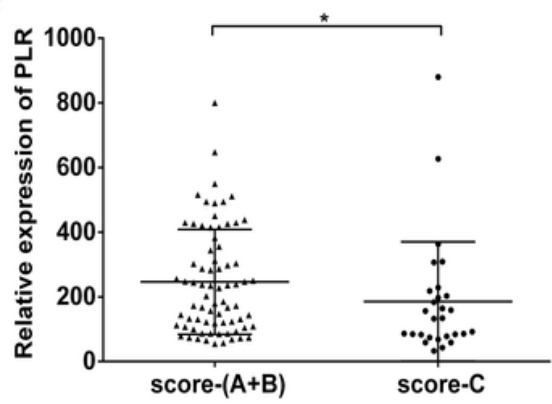

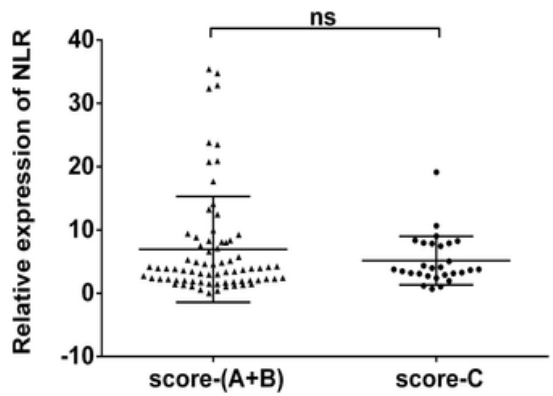

Figure 3

(A) The ROC curve analysis for the diagnostic value of MPV (AUC $=0.772,95 \% \mathrm{Cl}=0.708-0.836, \mathrm{p}<0.0001), \mathrm{RDW}(\mathrm{AUC}=0.970,95 \% \mathrm{Cl}=0.949-0.990, \mathrm{p}<$ $0.0001), \mathrm{MPV} / \mathrm{PC}$ ratio $(\mathrm{AUC}=0.876,95 \% \mathrm{Cl}=0.822-0.931, \mathrm{p}<0.0001), \mathrm{NLR}(\mathrm{AUC}=0.817,95 \% \mathrm{Cl}=0.755-0.879, \mathrm{p}<0.0001), \mathrm{PLR}(\mathrm{AUC}=0.702,95 \% \mathrm{Cl}=$ $0.626-0.778, p<0.0001)$ and model(AUC $=0.987,95 \% \mathrm{Cl}=0.975-0.999, \mathrm{p}<0.0001)$ in 102 patients with HBV-related cirrhosis.. (B) MPV/PC ratios of patients with HBV-related cirrhosis in different Child-Pugh scores $(p=0.001)$. (C) PLR of patients with HBV-related cirrhosis in different Child-Pugh scores ( $p=0.016)$. (D) MPV of patients with HBV-related cirrhosis in different Child-Pugh scores ( $p=0.632)$. (E) RDW of patients with HBV-related cirrhosis in different Child-Pugh scores $(p=0.549)$. (F) NLR of patients with HBV-related cirrhosis in different Child-Pugh scores $(p=0.848)$. ns $\llbracket$ no significance, ${ }^{\star} p<0.05,{ }^{\star *} p<0.01$. 

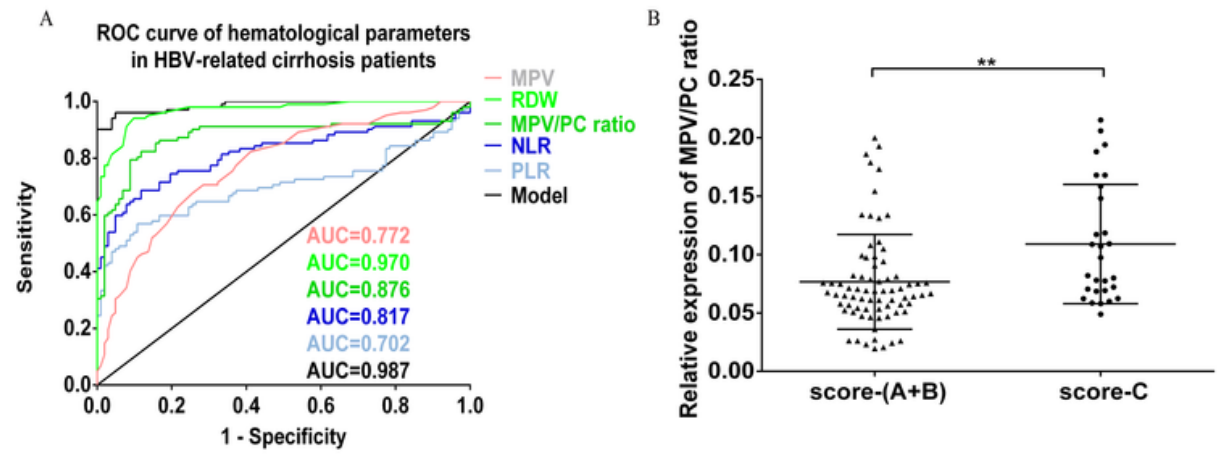

D

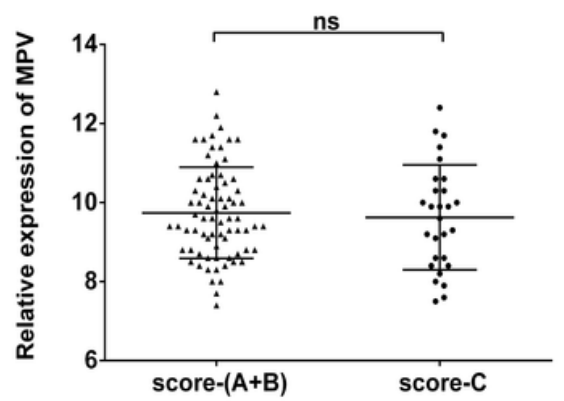

E

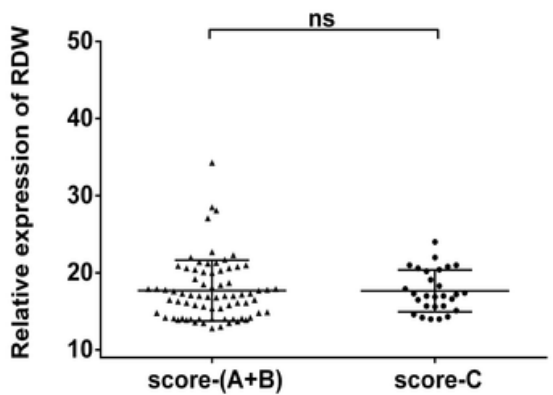

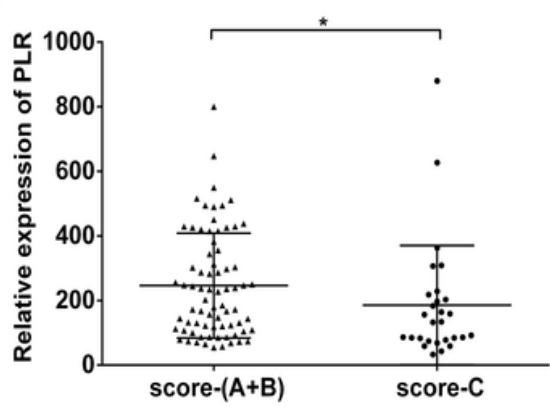

$\mathrm{F}$

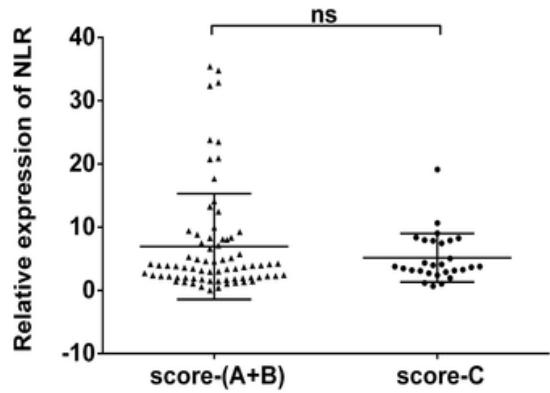

Figure 4

(A) The ROC curve analysis for the diagnostic value of MPV (AUC $=0.772,95 \% \mathrm{Cl}=0.708-0.836, \mathrm{p}<0.0001), \mathrm{RDW}(\mathrm{AUC}=0.970,95 \% \mathrm{Cl}=0.949-0.990, \mathrm{p}<$ $0.0001), \mathrm{MPV} / \mathrm{PC}$ ratio $(\mathrm{AUC}=0.876,95 \% \mathrm{Cl}=0.822-0.931, \mathrm{p}<0.0001), \mathrm{NLR}(\mathrm{AUC}=0.817,95 \% \mathrm{Cl}=0.755-0.879, \mathrm{p}<0.0001), \mathrm{PLR}(\mathrm{AUC}=0.702,95 \% \mathrm{Cl}=$ $0.626-0.778, p<0.0001)$ and model $(A U C=0.987,95 \% \mathrm{Cl}=0.975-0.999, \mathrm{p}<0.0001)$ in 102 patients with HBV-related cirrhosis.. (B) MPV/PC ratios of patients with HBV-related cirrhosis in different Child-Pugh scores $(p=0.001)$. (C) PLR of patients with HBV-related cirrhosis in different Child-Pugh scores ( $p=0.016)$. (D) MPV of patients with HBV-related cirrhosis in different Child-Pugh scores ( $p=0.632)$. (E) RDW of patients with HBV-related cirrhosis in different Child-Pugh scores $(p=0.549)$. (F) NLR of patients with HBV-related cirrhosis in different Child-Pugh scores $(p=0.848)$. ns \no significance, ${ }^{*} p<0.05,{ }^{* *} p<0.01$.
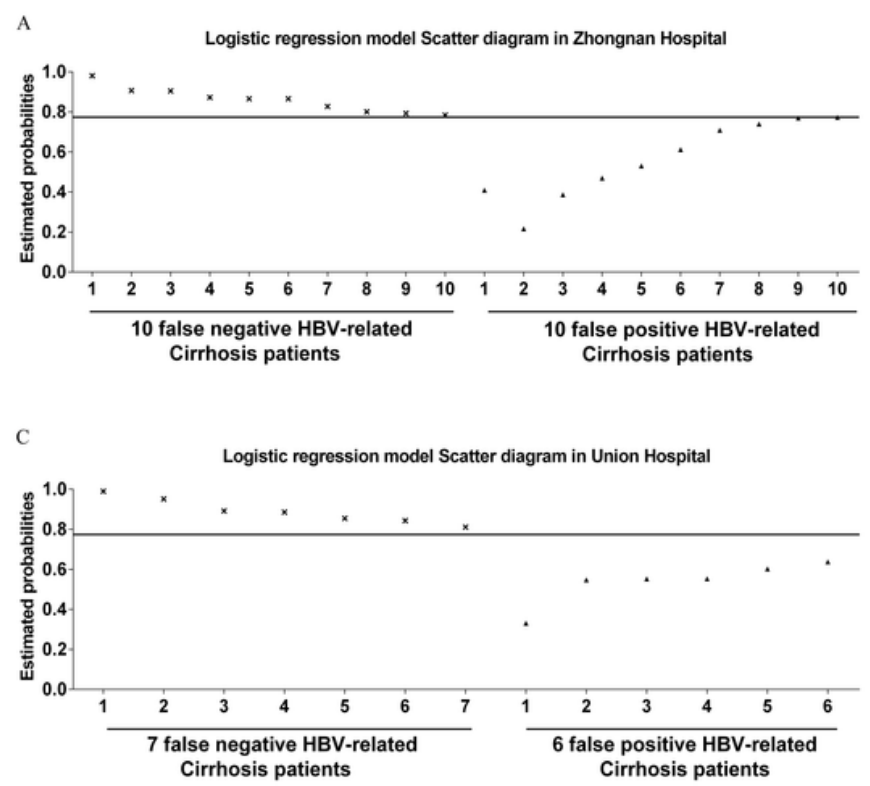
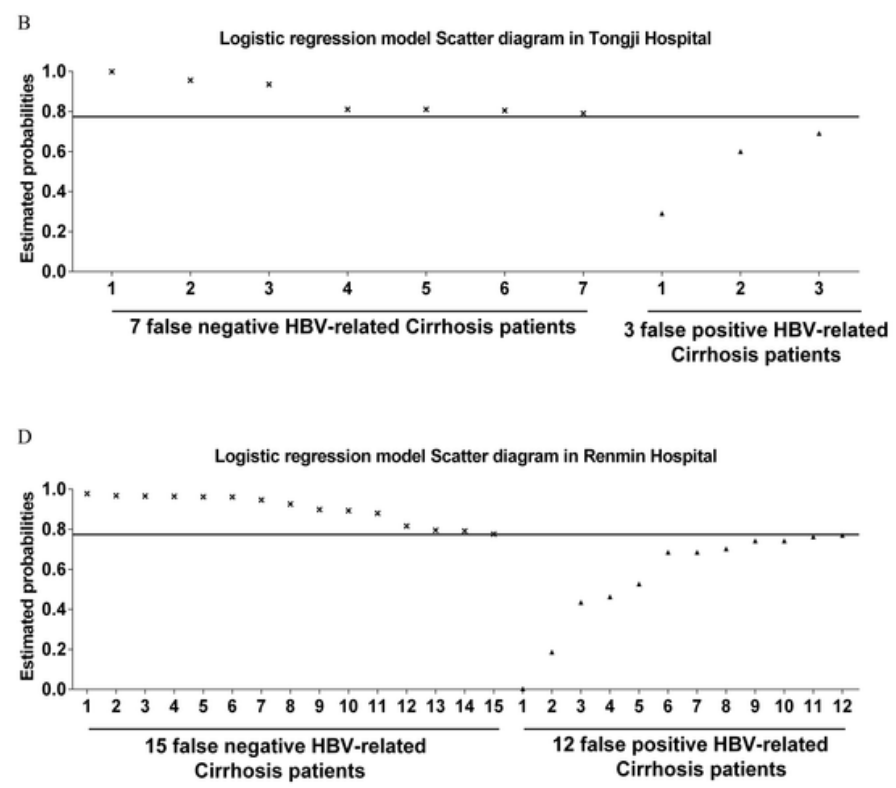

Figure 5

The scatter diagrams of the logistic regression model in four external validation sets. (A) 10 false negative patients with HBV-related cirrhosis and 10 false positive patients in the cohort of Zhongnan Hospital. (B) 7 false negative patients with HBV-related cirrhosis and 3 false positive patients in the cohort of Tongji Hospital. (C) 7 false negative patients with HBV-related cirrhosis and 6 false positive patients in the cohort of Union Hospital. (D) 15 false negative patients with HBV-related cirrhosis and 12 false positive patients in the cohort of Renmin Hospital 
A

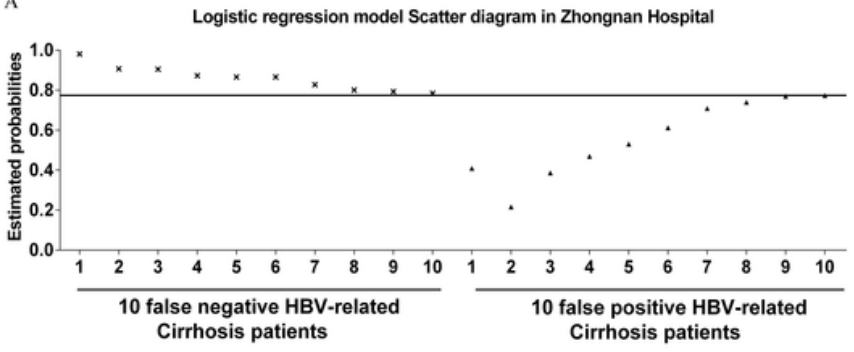

C

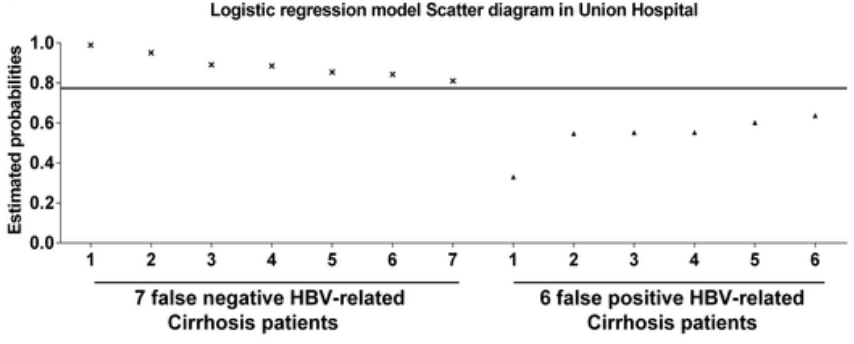

B

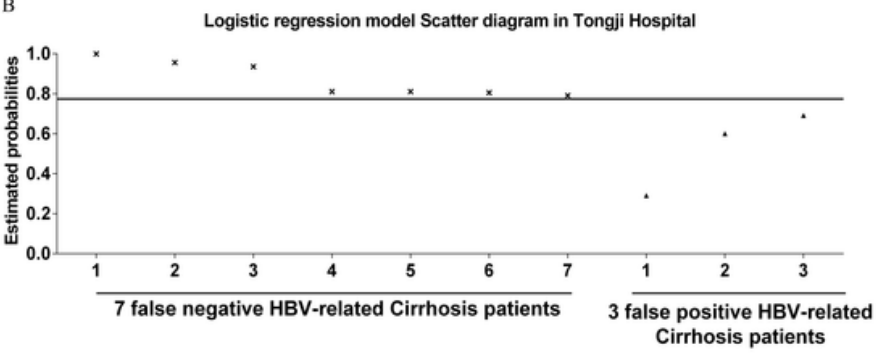

D

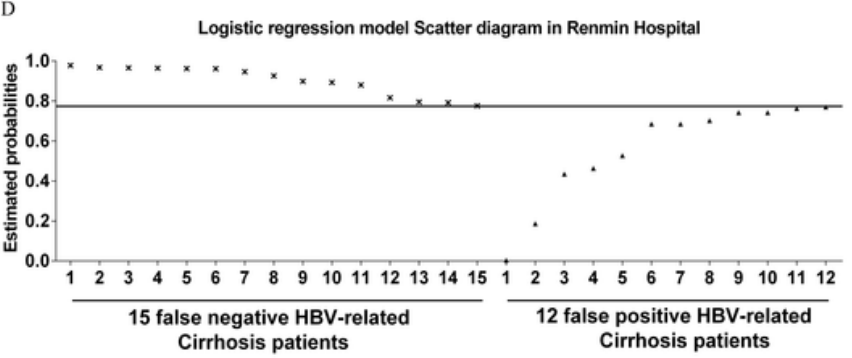

Figure 5

The scatter diagrams of the logistic regression model in four external validation sets. (A) 10 false negative patients with HBV-related cirrhosis and 10 false positive patients in the cohort of Zhongnan Hospital. (B) 7 false negative patients with HBV-related cirrhosis and 3 false positive patients in the cohort of Tongji Hospital. (C) 7 false negative patients with HBV-related cirrhosis and 6 false positive patients in the cohort of Union Hospital. (D) 15 false negative patients with HBV-related cirrhosis and 12 false positive patients in the cohort of Renmin Hospital
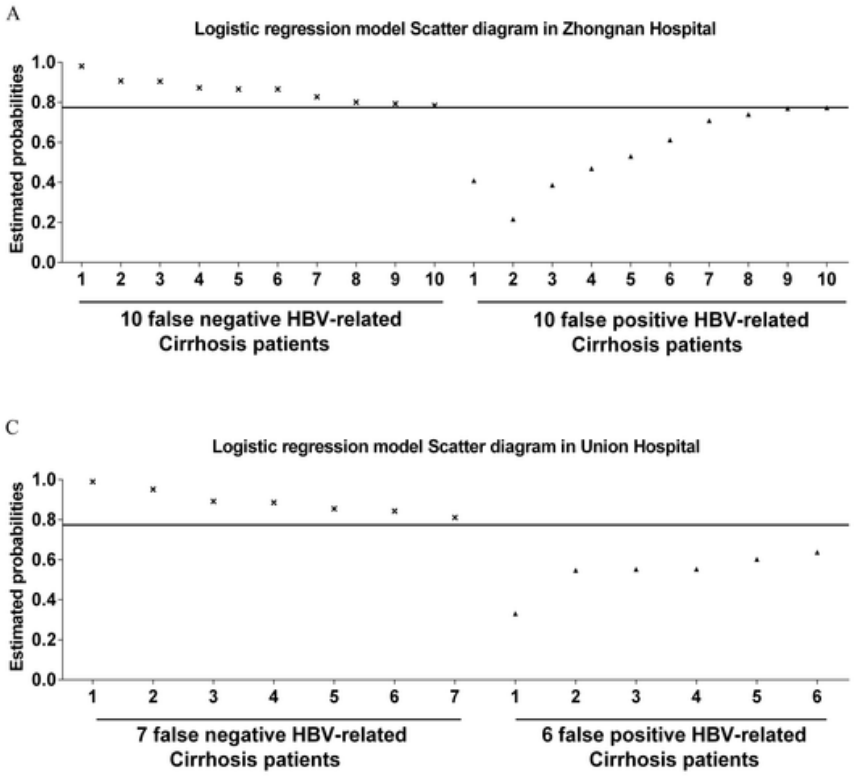

B
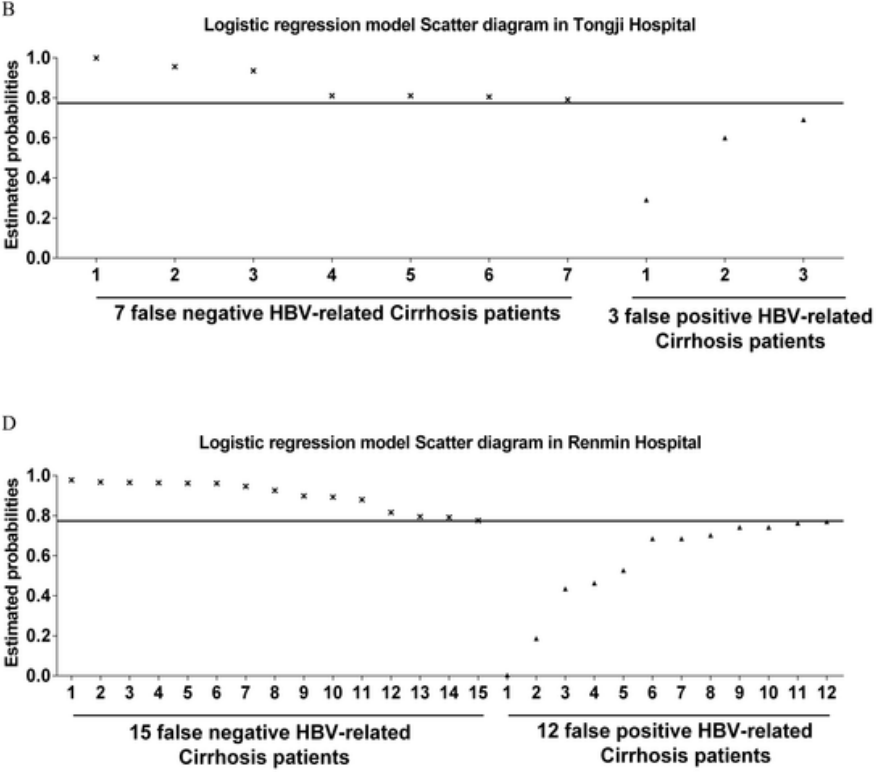

Figure 6

The scatter diagrams of the logistic regression model in four external validation sets. (A) 10 false negative patients with HBV-related cirrhosis and 10 false positive patients in the cohort of Zhongnan Hospital. (B) 7 false negative patients with HBV-related cirrhosis and 3 false positive patients in the cohort of Tongji Hospital. (C) 7 false negative patients with HBV-related cirrhosis and 6 false positive patients in the cohort of Union Hospital. (D) 15 false negative patients with HBV-related cirrhosis and 12 false positive patients in the cohort of Renmin Hospital

\section{Supplementary Files}

This is a list of supplementary files associated with this preprint. Click to download.

- Tables1andS2.docx 
- Tables1andS2.docx

Page 13/13 